assistance from a physician in order to benefit their patient. However, studies support that in only $23 \%$ of consultations was the patient allowed to complete their opening statement of concern, and in $69 \%$ of cases the physician interrupted the patient's statement to re-direct the enquiry to the physician's agenda. This IBD service recognised the paradigm in its approach to patient consultation. An innovative patient centred agenda sheet was developed In order to redesign the consultation model to allow patients the opportunity to put forward their concerns to be addressed during the consultation.

Methods A patient agenda sheet was developed. Page one included; an objective, rapid assessment tool, utilising the Bristol stool chart alongside an amalgamation of the Harvey Bradshaw index and Simple Clinical Colitis Activity Index. This allows the clinician to quickly establish disease activity and severity.

Page two is a free text section which includes suggestions for discussion points during the consultation which prompts the patient to address their concerns.

Patient agenda sheets were distributed when patients arrived in clinic to be completed in the waiting room. The sheets were used by the clinicians as a prompt during the consultation.

An audit of 100 clinic letters and patient agenda sheets was undertaken to establish emerging themes. The clinician perspective regarding the use of the patient agenda sheet was also analysed.

Results Uptake was positive with a 94\% completion rate.

There were six main themes identified included; medication issues, managing flare ups, fertility, travel, surveillance colonoscopy and general check-ups.

The agenda sheet reduced clinician time spent establishing disease activity and focused the consultation on addressing the patient's agenda.

Conclusion We successfully changed the consultation model from a physician's agenda to a patient centred approach. As well as empowering patients to discuss the issues that concern them, the patient agenda sheet has allowed clinicians to identify topics within the consultation which may not have previously addressed such as pregnancy and fertility. Furthermore it has facilitated rapid signposting to appropriate clinics such as Guided Self- management.

\section{PTH-091 EFFECTS OF VEDOLIZUMAB ON HRQOL AND WORK PRODUCTIVITY IN CROHN'S DISEASE: RESULTS FROM VERSIFY}

'S Danese, ${ }^{2} \mathrm{~S}$ Adsul, ${ }^{2} \mathrm{D}$ Lindner, ${ }^{3} \mathrm{~S}$ Jones, ${ }^{4} \mathrm{H}$ Patel, ${ }^{5} \mathrm{JF}$ Colombel. ${ }^{1}$ Humanitas University, Milan, Italy; ${ }^{2}$ Takeda Pharmaceuticals International AG, Switzerland; ${ }^{3}$ Takeda Development Centre Europe Ltd, London, UK; ${ }^{4}$ Takeda International, Deerfield, USA; ${ }^{5}$ /cahn, School of Medicine at Mount Sinai, USA

\subsection{6/gutjnl-2019-BSGAbstracts. 150}

Introduction The open-label, phase $3 \mathrm{~b}$, single-arm VERSIFY trial demonstrated that intravenous (IV) vedolizumab (VDZ) induced endoscopic healing in patients (pts) with moderately to severely active Crohn's disease (CD). We evaluated the effects of IV VDZ on quality of life (QOL) and work productivity over a 52-week study period.

Methods 56 patients were enrolled into an IV vedolizumab 52-week substudy. QOL was assessed using Inflammatory Bowel Disease Questionnaire (IBDQ) and Euro Quality of
Life-5D (EQ-5D) utility index and visual analogue scale (VAS), and work productivity using Work Productivity and Activity Impairment (WPAI-CD). For the 52-week substudy population $(n=56)$, changes over 52 weeks were evaluated. IBDQ remission was considered as a total IBDQ score of $\geq 170$ points, with an improvement of $\geq 16$ points considered clinically meaningful. Outcomes were examined by endoscopic remission status and by prior anti-tumour necrosis factor-alpha (anti$\mathrm{TNF} \alpha)$ use.

Results Mean pt age was 39.6 years, 54\% were male, 43\% had prior anti-TNF $\alpha$ treatment and 29\% achieved endoscopic remission at any ileocolonoscopy visit up to Week 52. Improvements in IBDQ total score were observed as early as Week 14 and were sustained up to Week 52. At week 52 improvements were greater in pts with endoscopic remission (183 vs 164 , table 1 ) and in pts with no prior anti-TNF $\alpha$ use (178 vs 157 , table 1 ). Similar trends of greater improvements in EQ-5D utility index (0.91 vs 0.83 ) and VAS (79 vs 68) were observed at Week 52 in pts with endoscopic remission. At Week 52, EQ-5D utility scores improved equally regardless of prior anti-TNF $\alpha$ use, whereas EQ-5D VAS scores were slightly higher in pts naïve to anti-TNF $\alpha$ vs those who had previously failed anti-TNF $\alpha$ treatment. Improvements in WPAI-CD subscores were consistently higher in pts with endoscopic remission; overall work impairment and daily activities impairment were substantially improved in pts naïve to antiTNF $\alpha$.

Conclusions Overall, IV VDZ treatment was associated with substantial improvements in both QOL instruments and work productivity measures. The improvements in QOL and work productivity were greater among pts who achieved endoscopic remission and pts who had no prior anti-TNFo treatment.

\begin{tabular}{lll}
$\begin{array}{l}\text { Abstract PTH-091 Table 1 } \\
\text { Week 52 }\end{array}$ & Changes in IBDQ from baseline to \\
\hline Instrument & Baseline (Week 0) & Week 52 \\
\hline IBDQ Total, mean (SD) [N] & & \\
Overall & $127.2(34.1)[55]$ & $169.2(35.3)[56]$ \\
Endoscopic remission - Yes & $120.1(35.2)[16]$ & $182.8(29.1)[16]$ \\
Endoscopic remission - No & $130.1(33.7)[39]$ & $163.7(36.5)[40]$ \\
Prior anti-TNF $\alpha$ use -Yes & $128.9(35.5)[24]$ & $157.1(39.1)[24]$ \\
Prior anti-TNF $\alpha$ use - No & $125.8(33.6)[31]$ & $178.3(29.7)[32]$ \\
\hline
\end{tabular}

\section{PTH-092 EARLY 'REAL WORLD' EXPERIENCE WITH TOFACITINIB FOR MODERATE TO SEVERE ULCERATIVE COLITIS}

Sailish Honap*, Esha Sharma, Shuvra Ray, Georgina Cunningham, Aravind Tamilarasan, Joel Mawdsley, Simon Anderson, Jeremy Sanderson, Peter Irving. Guy's and St. Thomas' Hospitals NHS Foundation Trust, London, UK

\subsection{6/gutjnl-2019-BSGAbstracts. 151}

Introduction Tofacitinib is an oral, small molecule Janus kinase inhibitor, which recently received NICE approval for the treatment of moderate to severe treatment refractory ulcerative colitis. We present early clinical and biochemical outcome data for a small group of new starters in a tertiary IBD referral centre. 
Methods A retrospective cohort analysis of patients was undertaken using prospectively maintained records. Patients commenced on tofacitinib through the patient access scheme between October 2018 to February 2019 were included. Clinical disease activity was measured at baseline, at four and eight weeks using the Simple Clinical Colitis Activity Index (SCCAI). Faecal calprotectin and C-reactive protein were measured at baseline and eight weeks.

Results At the time of submission, 16 patients had commenced tofacitinib, with outcome data available for 8 patients who had reached at least four weeks of treatment. All 8 patients (median age 46) with Mayo 2-3 colitis demonstrated on preinduction endoscopy, were previously exposed to an anti-TNF agent, of which 6 had also failed vedolizumab. Median baseline SCCAI $(n=8)$ fell from 8 (range 2-14) to $3(1-5)$ after four weeks and remained stable at eight weeks. Median baseline faecal calprotectin $(n=5)$ fell from $364(131-645)$ to 95 (30-289). One patient reaching week 16 was in endoscopic remission. Tofacitinib was well tolerated with only one patient reporting a mild headache and diarrhoea, which self-resolved in under a week. No haematological or biochemical abnormalities were noted.

Conclusions Our early experience with tofacitinib for moderate to severe ulcerative colitis is encouraging, with an improvement in SCCAI and faecal calprotectin in all our patients. Oral dosing and a quicker onset of action are other advantages, which may enable positioning above vedolizumab. Further real life data is necessary in this setting to demonstrate effectiveness and a longer term safety profile.

\section{PTH-093 EFFICACY OF THIOPURINE MONOTHERAPY IN THE UK INFLAMMATORY BOWEL DISEASE BIORESOURCE COHORT}

${ }^{1}$ You Yi Hong* ${ }^{*}$ 1,2Laetitia Pele, ${ }^{1,2}$ Rachel Simpkins, ${ }^{1,2}$ Catherine Thorbinson,
${ }^{1,2}$ Deepthy Francis, ${ }^{2}$ Rasha Shawky, 1,2Miles Parkes. 'Department of Medicine, University of
Cambridge, Cambridge, UK; ${ }^{2}$ IBD Bioresource, NIHR BioResource, Cambridge, UK

10.1136/gutjnl-2019-BSGAbstracts.152

Introduction IBD BioResource is currently recruiting patients with Crohn's Disease (CD), Ulcerative Colitis (UC) and IBDU from 89 hospitals UK-wide. To date $>19,000$ patients have been recruited with detailed clinical phenotype data plus serum and DNA, and all will have had Genome-Wide Association Scans \pm whole genome sequencing by April 2019. Subjects can be recalled by genotype or phenotype for downstream studies by any investigator.

Thiopurines play a key role in the management of IBD used either as monotherapy or in combination with other treatments to maintain remission. However, data regarding long term effectiveness are sparse.

Aims To characterise the long term effectiveness of thiopurine monotherapy among subjects in the IBD BioResource cohort

Method IBD phenotype data were extracted by research nurses and clinicians in each hospital site following case note review and uploaded to a Redcap database. Response to treatment was empirically classified as 'effective', 'not effective', and 5 other categories ('transient', 'partial', 'intolerant' etc.). By interrogating the Redcap database and analysing the data in $\mathrm{R}$ we sought to identify the proportion of patients in whom thiopurine was effective as monotherapy - in whom
(1) treatment was classified as 'effective' and (2) there had been no escalation to biologic therapy or need for surgery for the duration of thiopurine therapy. Patients started on anti TNF therapy at thiopurine initiation or undergoing surgery in the 1 year prior to initiation were excluded since we could not assess effectiveness of thiopurine monotherapy in these groups.

Results Data were available on 8296 IBD BioResource subjects $(48.3 \%$ male) treated with a thiopurine and meeting inclusion criteria for assessment. In 2417 patients (29.1\%) thiopurine monotherapy had been deemed to be an effective maintenance treatment - meeting both criteria (1) and (2) above. Long term effectiveness was higher in UC/IBDU $(1531 / 3485 ; 43.9 \%)$ compared to CD $(883 / 4799 ; 18.4 \%$ Chi Sq $\mathrm{P}<0.0001)$. 3459/8296 (41.7\%) treated with thiopurine were started within 1 year of diagnosis (Figure 1). Mean duration on drug $=5.9$ years at the time of IBD BioResource recruitment (at least $78 \%$ of those in whom thiopurine monotherapy has been effective are still on this therapy).

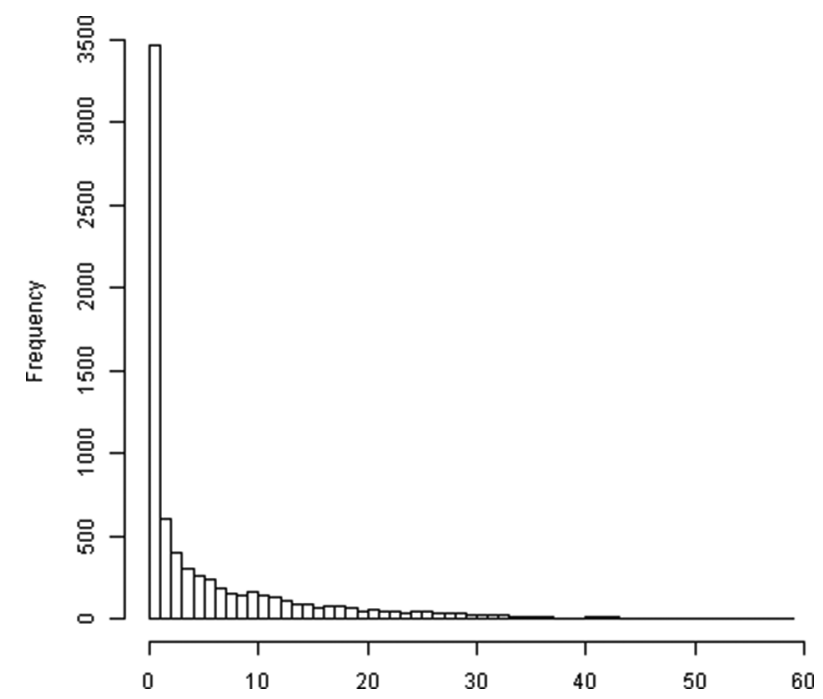

Abstract PTH-093 Figure 1 Time (years) from diagnosis to thiopurine initiation

Conclusion Thiopurines can be effective in producing durable remission, particularly in UC. Pharmacogenetic studies will follow. The IBD BioResource is open to all investigators for recall of well characterised patient cohorts.

\section{PTH-094 THIOPURINE ADVERSE EVENTS IN PATIENTS WITH INFLAMMATORY BOWEL DISEASE IN THE UK- IBD BIORESOURCE COHORT}

You Yi Hong*, Achini Diana Withanachchi, Rasha Shawky, Miles Parkes. Addenbrooke's Hospital, Cambridge, UK

\subsection{6/gutjnl-2019-BSGAbstracts.153}

Introduction The Inflammatory Bowel Disease (IBD) BioResource is recruiting patients with Crohn's Disease (CD), Ulcerative Colitis (UC) or IBD type Unclassified (IBDU) from 89 hospitals UK-wide. $>19,000$ subjects have been recruited to 